\title{
La coexistencia de problemas nutricionales en niños menores de 5 años en el Perú 2007-2010
}

Coexistence of nutritional disorders in children under the age of 5 in Peru 2007-2010

\author{
Jaime Pajuelo Ramírez ${ }^{1}$, Marianella Miranda Cuadros ${ }^{2}$ \\ 1 Profesor Principal y Miembro Permanente del Instituto de Investigaciones Clínicas. Facultad de Medicina, Universidad Nacional Mayor de \\ San Marcos, Lima, Perú. \\ ${ }^{2}$ Centro Nacional de Alimentación y Nutrición (CENAN), Instituto Nacional de Salud, Lima, Perú.
}

\begin{abstract}
Resumen
Introducción. La transición que vive el país es de tipo no occidentalizada, por cuanto persisten problemas que no han sido solucionados (deficiencias nutricionales) con otros emergentes (sobrepeso y obesidad). Objetivos. Describir la presencia de coexistencia de desnutrición crónica (DC) con obesidad (O), anemia nutricional (AN) y deficiencia de vitamina A (DVA), así como de obesidad (O) con AN y DVA en niños menores de 5 años. Diseño. Análisis secundario de datos del MONIN 2007-2010. Lugar. Estudio nacional. Participantes. Niños menores de 5 años. Intervenciones. Un total de 3764 niños fueron pesados y tallados. A 2808 niños se les dosó hemoglobina $(\mathrm{Hb})$ y a 1524 retinol sérico. Se consideró AN una Hb debajo de $11 \mathrm{~g} / \mathrm{dL}$. Se tomó en cuenta las correcciones por altitud. El punto de corte de DVA fue un retinol sérico < de $20 \mathrm{ug} / \mathrm{dL}$. Se estableció la DC y O con la relación talla/edad y peso/talla, con los niveles de corte de $\leq$ de $2 \mathrm{DE} y \geq$ de $2 \mathrm{DE}$, respectivamente, según la referencia de la OMS. El análisis fue con muestras complejas. Se calculó estadísticas descriptivas y regresión logística con IC del $95 \%$ y un nivel de significancia del $<0,05$. Resultados. El $22,4 \%$ de DC era anémico, $21,7 \%$ presentó DVA y 4,8\% obesidad. El ser anémico representó cuatro veces más riesgo de ser DC. El 5\% de obesos fue anémico y 4,2\% tuvo DVA; no se encontró asociación. Conclusiones. Se halló un escenario más complejo del que representan los problemas en forma individual, planteando un reto para las personas que tienen la responsabilidad de diseñar las políticas nutricionales, ya que amerita una nueva visión en el enfrentamiento de esta problemática, en especial en los Programas de Asistencia Alimentaria.
\end{abstract}

Palabras clave. Problemas Nutricionales; Niños Pre-escolares.

\section{Abstract}

Introduction. Our country's transition is the non-westernized type, because problems that have not been solved persist (nutritional deficiencies) along with emerging problems (overweight and obesity). Objective. To describe the presence of coexisting chronic malnutrition (CM) with obesity (O), nutritional anemia (NA) and vitamin A deficiency (VAD) and obesity (O) with NA and VAD in children under the age of 5. Design. Secondary analysis of the MONIN 2007-2010 survey. Setting. National study. Participants. Children under the age of 5. Interventions. A total of 3764 children were weighed and measured. Hemoglobin dosage was obtained in 2808 children, serum retinol in 1524 . Hb below $11 \mathrm{~g} / \mathrm{dL}$ was diagnosed as NA. Correction by altitude was taken into account. The cutoff for VAD was serum retinol $<20 \mathrm{ug} / \mathrm{dL}$. The relation of $\mathrm{CM}$ and $\mathrm{O}$ with height/age ratio and weight/height, with cutting levels $\leq 2 \mathrm{SD}$ and $\geq 2$ $\mathrm{SD}$, respectively, were done according to the WHO reference. Analysis used complex samples, descriptive statistics and logistic regression, calculated with a $95 \%$ confidence interval and a significance level of $<0.05$. Results. $22.4 \%$ of CM were anemic, $21.7 \%$ presented VAD and $4.8 \%$ obesity. The risk of being anemic represented four times higher than the risk of being CM. $5 \%$ of the obese were anemic and $4.2 \%$ had VAD; no association was found. Conclusions. We found a scenario more complex than the one problems represent at an individual level. This poses a challenge to the people in charge of designing nutritional policies, since this deserves a new vision in the management of this issue, especially in the Food Assistance Programs.

Keywords. Nutritional Problems; Pre-school Children.

An Fac med. 2016;77(4):345-9 / http://dx.doi.org/10.15381/anales.v77i4.12650

\section{INTRODUCCIÓN}

Los principales problemas nutricionales que afectan a los niños peruanos menores de 5 años son la desnutrición crónica (DC), la obesidad (O) y la deficiencia de micronutrientes, específicamente los que se refieren a la anemia nutricional (AN) y la vitamina A (DVA). La presencia de estas enfermedades conlleva a una serie de complicaciones que comprometen la salud futura de los niños. Es bien conocido que la nutrición en los niños tiene rol importante en el desarrollo físico y mental y que esto repercute de alguna manera en las condiciones socioeconómicas de un país.

Estas patologías tienen una presencia y tendencias diferentes. La historia muestra que la direccionalidad que han seguido la DC y la O son completamente opuestas, mientras la primera tiende a disminuir todo lo contrario sucede con la segunda. En lo que respecta a la DC, la prevalencia ha pasado de $27,8 \%$ en el año $2007^{(1)}$ hasta $14,4 \%$ en el $2015^{(2)}$, mientras que la $\mathrm{O}$ se ha incrementado de $4 \%$ en $1984{ }^{(3)}$ a $6,5 \%$ en el año $2008{ }^{(4)}$. La AN ha disminuido de $45 \%$ en el año $1975^{(5)}$ a 35,6\% en el $2015^{(2)}$. Es necesario remarcar que en estos últimos años no ha variado sustantivamente; incluso las prevalencias 
se han incrementado ligeramente. En cuanto a la DVA se ha mantenido alrededor de $11 \%{ }^{(6)}$. En base a estos resultados se puede afirmar que el problema de mayor magnitud que afecta los niños peruanos es la AN, seguido de la DC, DVA y $\mathrm{O}$.

La transición que está sufriendo el mundo es un proceso en el campo epidemiológico, demográfico y nutricional y que se manifiesta con distintas velocidades en los diferentes países, incluyendo el Perú. Este fenómeno ha hecho que los problemas mencionados que se percibían de manera independiente tengan que verse de una manera más colectiva, dado que es posible que puedan coexistir. Las coexistencias pueden darse en un mismo niño, en el hogar y en la misma área geográfica, lo que de alguna forma podrían potencializar el daño que puedan ocasionar ${ }^{(7)}$.

En el Perú se han publicado varios estudios donde se aprecia la presencia de la coexistencia. En ese sentido, uno de los primeros trabajos donde se muestra a la $\mathrm{DC}$ y la $\mathrm{O}$ en un mismo niño fue realizado con beneficiarios de Programas de Asistencia Alimentaria ${ }^{(8)}$; posteriormente se agregó el problema de la AN ${ }^{(9-10)}$. Dentro de la misma área geográfica, la presencia de la $\mathrm{O}$ y la DC ocurre de diferente manera. Hay dominios como Lima Metropolitana, costa norte, centro y sur donde hay mayor prevalencia de $\mathrm{O}$ frente a la $\mathrm{DC}$, mientras que en otros como sierra norte, centro, sur y selva prima la DC ${ }^{(11)}$. Todos estos estudios fueron realizados en niños de 6 a 9 años.

El objetivo del presente estudio es describir la presencia de coexistencia de la DC con la O, la AN y la DVA, así como de la O con la AN y la DVA en niños menores de 5 años en el Perú.

\section{METODOLOGÍA}

El presente estudio proviene de un análisis secundario de los datos del Monitoreo Nacional de Indicadores Nutricionales (MONIN) recabados en el periodo 2007-2010. El MONIN es una encuesta continua (transversal repetida), por muestreo aleatorio multietápico, del universo de niños menores de cinco años y gestantes residentes en el Perú, dividido en 5 ámbitos geográficos y 6 periodos aproximadamente trimestrales (semanas permutadas aleatoriamente que fueron $78 \%$ del tiempo entre el 03-noviembre-2008 y el 02-abril-2010). MONIN calculó la muestra para obtener un margen de $95 \%$ de confianza de 7,5 a $15 \%$ para proporciones de prevalencia entre 20 a $80 \%$ (efecto de diseño de 1,5 ) en cualquier trimestre.

A 3764 niños menores de 5 años se los midió; a 2808 niños se les dosó hemoglobina y a 1524 retinol sérico. A los niños se les pesó y talló de acuerdo a metodología internacionalmente aceptada, incluida en la Guía elaborada por el Centro Nacional de Alimentación y Nutrición (CENAN). Se utilizaron balanzas digitales con una precisión de $100 \mathrm{~g}$ y tallímetros confeccionados de acuerdo a las especificaciones técnicas del CENAN ${ }^{(12)}$.

El diagnóstico de $\mathrm{DC}$ y $\mathrm{O}$ se hizo mediante la relación talla/edad y el peso/talla, respectivamente, tomando como criterio diagnóstico el menor de menos dos desvío estándar y de mayor de más dos desvío estándar. Para ambos casos se utilizó la población referencial de la Organización Mundial de la Salud (OMS) ${ }^{(13)}$.
Los análisis bioquímicos realizados fueron hemoglobina $(\mathrm{Hb})$ usando el sistema Hemo-Cue y retinol sérico mediante la obtención de una muestra de sangre venosa. Los puntos de corte para diagnosticar $\mathrm{AN}$ fue una $\mathrm{Hb}$ por debajo de $11 \mathrm{~g} / \mathrm{dL}^{(14)}$. Se realizó las respectivas correcciones por la altitud ${ }^{(15)}$. El punto de corte para determinar DVA fue un retinol sérico $<$ de $20 \mathrm{ug} / \mathrm{dL}^{(16)}$.

El análisis estadístico se hizo mediante muestras complejas utilizando el paquete estadístico de SPSS Inc. SPSS Complex Samples 15.0 Chicago, IL. SPSS 2006. Los datos son presentados en prevalencias, con su respectivo IC al 95\%. La asociación de las variables categóricas se hizo mediante la prueba de chi cuadrado con un $\alpha<0,05$.

En el caso de la normalidad, se aplicó la prueba de Kolgomorov-Smirnov y para la homogeneidad de las varianzas la prueba de Levene.

Se estimó análisis de regresión logística múltiple para verificar asociación entre factores determinantes. Los OR fueron ajustados.

\section{RESULTADOS}

En la tabla 1 se muestra la prevalencia de $\mathrm{DC}$ en relación al estatus de la $\mathrm{Hb}$ (no anémicos y anémicos), de la vitamina A (no deficientes y deficientes) y $\mathrm{O}$ (no obesos y obesos). Los grupos que

Tabla 1. Prevalencia de desnutrición crónica en niños menores de 5 años, según estado nutricionales.

\begin{tabular}{|c|c|c|c|c|c|c|c|}
\hline & & Número & $\%$ & IC & $p$ & $\mathrm{OR}$ & IC \\
\hline \multirow[t]{3}{*}{ Hemoglobina } & & & & & 0,001 & & \\
\hline & No anémico & 1843 & 17 & 15,4 a 19 & & 1 & \\
\hline & Anémico & 965 & 22,4 & 20 a 24,9 & & 3,8 & 3,1 a 4,7 \\
\hline \multirow[t]{3}{*}{ Vitamina A } & & & & &, 233 & & \\
\hline & No deficiente & 1358 & 17,9 & 16,1 a 20 & & 1 & \\
\hline & Deficiente & 166 & 21,7 & 15,7 a 28,3 & & 0,9 & 0,6 a 1,5 \\
\hline \multirow[t]{3}{*}{ Obesidad } & & & & & 0,001 & & \\
\hline & No obeso & 3518 & 19,2 & 18 a 20,5 & & 1 & \\
\hline & Obeso & 228 & 4,8 & 2,2 a 7,9 & & 2 & 0,6 a 6,6 \\
\hline
\end{tabular}


Tabla 2. Prevalencia de obesidad en niños menores de 5 años, según estados nutricionales.

\begin{tabular}{llcccccc} 
& & Número & $\%$ & IC & p & OR & IC \\
Hemoglobina & & & & & 0,27 & & \\
& No anémico & 1843 & 6 & 4,9 a 7,1 & & 0,1 & 0,05 a 1,1 \\
& Anémico & 965 & 5 & 3,5 a 6,4 & & 1 & \\
Vitamina A & & & & & 0,74 & & \\
& No deficiente & 1358 & 4,8 & 3,7 a 5,9 & & 1,3 & 0,5 a 3,6 \\
& Deficiente & 166 & 4,2 & 1,2 a 7,8 & & 1 & \\
\hline
\end{tabular}

presentaron las mayores prevalencias en orden descendente fueron los anémicos $(22,4 \%)$, los deficientes de vitamina A $(21,7 \%)$ y los obesos $(4,8 \%)$. Solo se encontró asociación de la DC con las variables de $\mathrm{Hb}(\mathrm{p}<0,001)$ y obesidad $(<0,001)$. El ser anémico representó un riesgo 4 veces mayor de tener DC que ser no anémico (OR 3,8 IC95\% 3,1 a 4,7).

En la tabla 2 se observa la prevalencia de $\mathrm{O}$ en relación al estatus de la $\mathrm{Hb}$ (no anémicos y anémicos) y de la vitamina A (no deficientes y deficientes). Las mayores prevalencias las presentaron los no anémicos (6\%) y los no deficientes de vitamina A $(4,8 \%)$, aunque estas diferencias no fueron significativas $(\mathrm{p}<0,05)$. El 5\% de obesos tenía AN mientras que $4,2 \%$ presentaba DVA. No se encontró asociación entre las variables.

\section{DISCUSIÓN}

Frente a la problemática nutricional que afecta a los niños, los diferentes gobiernos han diseñado políticas y estrategias para enfrentarlos. Estas han sido realizadas en función de las prevalencias presentadas por cada una de ellas. En la actualidad, y como producto de la transición, la presencia de coexistencias en nuestro país se caracteriza por presentar problemas que aún no han sido resueltos, con otros que recién están emergiendo, lo que obliga a hacer un replanteamiento de las mismas. El presente trabajo muestra esta realidad que se observa en el mismo individuo y en niños menores de 5 años.
En el presente estudio se ha encontrado que $4,8 \%$ de $\mathrm{DC}$ son $\mathrm{O}$; prevalencias más o menos similares se han hallado en niños hispanos en el NHANES 2003-2010 (17). En México, la prevalencia es de $5,9 \%$ en la población no indígena y $12,1 \%$ en los indígenas ${ }^{(18)}$ En Guatemala en la población no indígena es $1,2 \%$ frente a la indígena $2,8 \%^{(19)}$. En el Uruguay es $1,9 \%{ }^{(20)}$.

Existen hipótesis que tratarían de explicar este acontecimiento y una posibilidad es que la pobre nutrición en la infancia puede alterar los caminos fisiológicos que incrementan la posibilidad de convertirse en obesos después. Esta pobre nutrición fomenta de alguna manera un fenotipo ahorrador que va a aumentar la eficiencia del depósito de grasa ${ }^{(21)}$.

Algunos autores barajan la posibilidad de las dietas adecuadas o altas en energía pero pobres en micronutrientes tienen responsabilidad en el crecimiento lineal, como el zinc y en menor medida la vitamina A y el hierro, por ejemplo ${ }^{(22)}$. Golden describió dos tipos de deficiencia de nutrientes, el primero (hierro y vitamina A) que resulta en una depleción de los depósitos del cuerpo, pero que mantiene la línea de crecimiento; y el tipo 2 (zinc y proteínas) que resultan en una disminución de la línea de crecimiento, y todo esto asociado a la pobreza ${ }^{(23)}$.

Otros sugieren que la dieta ingerida es reducida en los nutrientes esenciales requeridos para el crecimiento, pero no para un incremento en el tejido adiposo; y que la 'programación nutricional' puede incidir en ciertos efectos hormo- nales que limitan el crecimiento lineal, pero que de ninguna manera afectan el potencial para ganar peso ${ }^{(24)}$.

En el Brasil, en poblaciones que se encuentran bajo los efectos de la transición, la obesidad puede estar ocurriendo como una secuela de la malnutrición en los primeros años de la vida y se especula que esta malnutrición sería debida a un balance negativo de energía. Esta situación a su vez puede promover una tendencia hacia el balance positivo de energía cuando se mejora la capacidad de disponer de alimentos. Por ejemplo, cuando el incremento de la edad permite la mayor capacidad de acceso de los alimentos vía el beneficio de la ayuda alimentaria o de mejoras en la complementación ${ }^{(25)}$. Dentro de esta misma línea de pensamiento, en China se señala que la coexistencia de DC y obesidad en el mismo niño, resulta de la malnutrición de proteínas y energía que retarda el crecimiento, a pesar del aumento del peso corporal ${ }^{(26)}$.

En cuanto a la relación de la DC con la AN se ha visto que entre los anémicos un 22,4\% tiene DC, mientras que en los no anémicos es en $17 \%$. Estas variables presentan una asociación significativa y prácticamente hay cuatro veces más riesgo de ser DC de los anémicos frente a los no anémicos (OR 3,98, IC95\% 3,1 a 4,7). En Sri Lanka han mostrado que a mayor DC, los promedios de $\mathrm{Hb}$ son menores en relación a los niños que presentan un crecimiento normal ${ }^{(27)}$. Situación parecida se señala en la India ${ }^{(28)}$.

La presencia de DC con la DVA se hace mayor en el grupo de deficientes $(21,7 \%)$ que en los normales $(17,9 \%)$. Algunos estudios muestran estas diferencias, como el de Faber, en Sur África, que encontró valores de 15,7 y $8,7 \%$, respectivamente ${ }^{(29)}$, y el de Amaya-Castellanos en Venezuela con $21,9 \%{ }^{(30)}$. Otros estudios muestran que los promedios de vitamina A son menores en los DC que en los normales ${ }^{(27)}$. En Brasil es 1,3\% (31).

Tanto la DC con la AN y con la DVA estarían condicionadas por el 
deficiente ingreso de alimentos, y por ende el de micronutrientes y episodios de diarrea que presentan los niños.

En la relación de la $\mathrm{O}$ con las deficiencias de micronutrientes del estudio, no se ha encontrado asociación alguna. Para el caso de la $\mathrm{O}$ y la AN, la prevalencia fue $5 \%$, parecida a la encontrada en hispanos en EE UU que fue $7 \%{ }^{(17)}$. En Guatemala, el 1,4\% presenta esta coexistencia, siendo menor en su población indígena $1,1 \%$ que en la no indígena $1,7 \%^{(19)}$. Manios demuestra que los obesos presentan un alto riesgo de tener anemia por deficiencia de hierro que sus pares con un estado nutricional normal ${ }^{(32)}$. Incluso algunos estudios consideran que un peso aumentado es significativo predictor del estatus del hierro ${ }^{(33)}$.

Algunos mecanismos han sido propuestos para explicar esta relación, principalmente como una inadecuada ingestión de hierro, un incremento en su requerimiento como consecuencia de una masa corporal aumentada y un incremento en el volumen sanguíneo ${ }^{(33)}$. Por otro lado, se ha demostrado que el estado inflamatorio que caracteriza a la obesidad perjudica la absorción y utilización del hierro debido a un incremento de la hepcidina, que es un regulador de la homeostasia del hierro, cuya producción es estimulada por los marcadores inflamatorios ${ }^{(34)}$. Además de la sobreproducción de hepcidina hay que tener en cuenta el lipocalin, por cuanto ambos conducen a un secuestro del hierro en el sistema reticuloendotelial ${ }^{(35)}$. Esta hipótesis se vio reforzada con lo que se observó que en los pacientes sometidos a cirugía bariátrica; mejoró su estatus de hierro ${ }^{(36)}$.

Asimismo estudios de intervención muestran que en los niños obesos anémicos la respuesta a la suplementación del hierro por vía oral se ha visto reducida ${ }^{(37)}$. Este detalle habría que tomarlo en cuenta, ya que dar sulfato ferroso es obligatorio en los niños menores de 5 años.

En lo que corresponde a la $\mathrm{O}$ y la vitamina A, la prevalencia de DVA encontrada es baja. Otros autores encontraron mayores prevalencias, como de Souza de 10\% (38), Sarni 18,6\% (39) y Teske $9,8 \%{ }^{(40)}$. En ninguna de ellas se encontró asociación. Se ha mencionado que el exceso de grasa tiene un papel de 'secuestrador' de las vitaminas liposolubles (A, D, E, K). Esto se hace más evidente cuando se trata de los $\square$ carotenoides y los $\square$ tocoferoles, donde sí hay una relación inversa con la obesidad ${ }^{(41)}$. Dado que la función de los antioxidantes de estos últimos, es que se genera la relación de la deficiencia de micronutrientes con las enfermedades crónicas no transmisibles.

En conclusión, estamos en presencia de un escenario mucho más complejo del que representan los problemas en forma individual. La presencia de las diferentes coexistencias entre las patologías nutricionales plantea un reto para las personas que tienen la responsabilidad de diseñar las políticas nutricionales, ya que amerita una nueva visión en el enfrentamiento de esta problemática, en especial en los Programas de Asistencia Alimentaria.

\section{REFERENCIAS BIBLIOGRÁFICAS}

1. Instituto Nacional de Estadística e Informática (INEI). Encuesta Demográfica y de Salud Familiar (ENDES 2007).

2. Instituto Nacional de Estadistica e Informática (INEI). Encuesta Demográfica y de Salud Familiar (ENDES 2015).

3. Ministerio de Salud. Instituto Nacional de Estadistica (INE). Encuesta Nacional de Nutrición y Salud 1984. Lima, 1984.

4. Pajuelo J, Miranda M, Campos M, Sánchez-Abanto J. Prevalencia de sobrepeso y obesidad en niños menores de cinco años en el Perú 2007-2010. Rev Peru Med Exp Salud Publica. 2011;28(2):169-72.

5. Pajuelo J, Amemiya I. Anemia nutricional en la población infantil del Perú. Rev Med Per. 1992;347:51-5.

6. Pajuelo J, Miranda M, Aguero R. Prevalencia de deficiencia de vitamina $A$ y anemia en niños menores de cinco años en el Perú. Rev Peru Med Exp Salud Publica. 2015;32(2):245-51.

7. Rivera J, Pedraza L, Martorell R, Gil A. Introduction to the double burden of undernutrition and excess weight in Latin America. Am J Clin Nutr. 2014;100(suppl):1613S-6S

8. Pajuelo J, Villanueva M, Chavez J. La denutrición crónica, el sobrepeso y la obesidad en niños de áreas rurales del Perú. An Fac med. 2000;61(3):201-6.

9. Pajuelo J, Vergara G, De la Cruz G. Coexistencia de problemas nutricionales en niños de 6 a 9 años de edad, de Centros Educativos de Matucana, Santa Eulalia y Lima. An Fac med. 2001;62(4):312-6.

10. Aparco J. Coexistencia de indicadores de malnutrición en escolares beneficiarios del subprograma escolar desayunos del Programa Nacional de Asistencia Alimentaria (PRONAA). Bol Inst Nac Salud. 2015;21(10-12):174-80.

11. Pajuelo J, Sánchez-Abanto J, Alvarez D, Tarqui C, Agüero R. Sobrepeso, obesidad y desnutrición crónica en niños de 6 a 9 años en el Perú. Rev Peru Med Exp Salud Publica. 2013;30(4):583-9.

12. Ministerio de Salud. Instituto Nacional de Salud (INS). Centro Nacional de Alimentación y Nutrición (CENAN). La Medición de la Talla y el Peso. Guía para el personal de salud del primer nivel de atención. Lima: INS/CENAN; 2004.

13. World Health Organization. Physical status: the use and interpretation of anthropometry. Report of a WHO Expert Committee. W H O Tech Rep Ser. 1995(854).

14. Organización Mundial de la Salud (OMS). Catálogo ISBN 3-906412-33-4. Ginebra 2007.

15. Center for Disease Control (CDC). Criteria for anemia in children and childbearing aged women. Morbidity and Mortality Weekly Report. 1989;38:400-4.

16. World Health Organization (WHO). Indicators for assessing vitamin A deficiency and the applications in monitoring and evaluating interventions programs. Geneva 1993

17. Iriart C, Bousan B, Rodriguez C, Handal A. Obesity and malnutrition among hispanic children in the US: double burden on health inequities. Rev Panam Salud Public. 2013;34(4).

18. Fernald L, Neufeld L. Overweight with concurrent stunting in very young children from rural Mexico: prevalence and associated factors. Eu J Clin Nutr. 2007;61:623-32.

19. Ramírez-Zea M, Kroker-Lobos M, Close-Fernandez $\mathrm{R}$, Canter R. The double burden of malnutrition in indigenous and nonindigenous Guatemala populations. Am J Clin Nutr. 2014;100(suppl):1644S$1651 \mathrm{~S}$.

20. Severi C, Moratorio X. Double burden of undernutrition and obesity in Uruguay. Am J Clin Nutr. 2014;100(suppl):1659-62.

21. Tzioumis E, Adair L. Childhood dual burden of under-and-over nutrition in low-and-middleincome countries: a critical review. Food Nut Bull. 2014;35(2):230-43.

22. Rivera J, Hotz C, Gonzalez-Cossio T, Neufeld L, Garcia-Guerra A. The effect of micronutrient deficiencies on child growth a review of results from Community-Based Supplementation Trials J Nutr. 2003;133:4010-20.

23. Golden M. Specific deficiencies versus growth failure: Type I and Type II nutrients. J Nutr Environmental Med. 1996;6:301-8.

24. Popkin B, Richards M, Montiero C. Stunting is associated with overweight in children of four nations that are undergoing the Nutrition. J Nutr. 1996;126:3009-16.

25. Sawaya A, Roberts S. Stunting and future risk of obesity: principal physiological mechanisms. Cad Saude Publica Rio de Janeiro. 2003;19(S1):S21S28.

26. Zong XN, Li H. Physical growth of children and adolescents in China over the past 35 years. Bull World Health Organ. 2014;92:555-64.

27. Marasinghe E, Chackrewarthy S, Abeysena Ch, Rajindrajith S. Micronutrients status and its relationship with nutritional status in preschool children in urban Sri Lanka. Asia Pac J Clin Nutr. 2015;24(1):144-51. doi:10.6133/apjcn.2015.24.1.17. 
28. Awasthi S, Das R, Verma T, Vir S. Anemia and undernutrition among preschool children en Uttar Pradesh, India. Indian Pediatrics. 2003;40:985-90.

29. Faber M, van Jaarsveld P, Kunneke E, Kruger S, Schoeman S, van Stuijvenberg M. Vitamin A and anthroprometic status of South African preschool children from four areas with known distinct eating patterns. Nutrition. 2015;31:64-71. Dx.doi. org/10.1016/j.nutr.2014.04.024.

30. Amaya-Castellanos D, Viloria-Castejón H, Ortega P, Gámez G, Urrieta J, Lobo P, Estévez J. Vitamin A deficiency and the anthropometric nutritional status of urban and rural marginalized children in the state of Zulia, Venezuela. Invest Clin. 2002;43(2):89-105.

31. Monteiro CW. Nutrition transition and double burden of undernutrition and excess of weight in Brazil. Am J Clin Nutr. 2014;100(suppl):1617S-1622S.

32. Manios Y, Moschonis G, Chrousos G, Lionis C, Mougios V, Kantilafti M, et al. The double burden of obesity and iron deficiency on children and adolescent in Greece: the Healthy Growth Study. J Hum Nutr Diet. 2013;26(5):470-8. doi:10.111/jhn.12025.

33. Yanoff L, Menzie C, Denkinger B, Sebring $\mathrm{N}, \mathrm{McHugh} \mathrm{T}$, et al. Inflammation and iron deficiency in the hypoferrinemia of obesity. Int J Obes (Lond). 2007;31:1412-9
34. Mc Cloung J, Karl J. Iron deficiency and obesity: the contribution of inflammation and diminished iron absorption. Nutr Rev. 2009;67:100-4.

35. Nikoronov A, Skalnaya M, Tinkov A, Skalny A. Mutual interaction between iron homeostasis and obesity. J Trace Elements Med Biol. 2015;30:20714. doi.org/10.1016/j.jtemb.2014.05.005.

36. Anty R, Dahman M, lanelli A, et al. Bariatric surgery can correct iron depletion in morbidly obese women: a link with chronic inflammation. Obes Surg. 2008;18:709-14.

37. Hutchinson C. A review of iron studies in overweight and obese children and adolescents: a double burden in the young? Eu J Nutr. 2016;16:1-9. doi. org/10.1007/s00394-016-1155-7.

38. De Souza L, Valeria da Veiga G, Ramalho R. Association of serum concentrations of retino and carotenoida with overweight in children and adolescents. Nutrition. 2007,23(5):392-7. Doi. org/10.1016/jnut2007.02.009.

39. Sarni R, Suano de Souza F, Ramalho R, de Olivera D, Kochi C, Catherino .P et al. Serum retinol and total carotene concentrations in obese pre-school children. Med Sci Monit. 2005;11(11):CR510-4.

40. Teske M, Melges P, de Souza I, Fonseca F, Sarni R. Plasma concentrations of retinol in obese children and adolescents: relationship to metabolic syndrome components. Rev Paul Pediatr. 2014;32(1):50-4.

41. Morinobu T, Murata T, Takaya R, Tamai $\mathrm{H}$. Nutritional status of $\beta$ carotene, $\alpha$ tocoferol and retinol in obese children. Int $\mathrm{J}$ Vitamin Nutr Res. 2002;72(3):119-23.

Artículo recibido el 4 de agosto de 2016 y aceptado para publicación el 10 de octubre de 2016.

Conflicto de interés: No existe conflicto de interés alguno.

Fuente de financiamiento: propio.

Correspondencia:

Dr. Jaime Pajuelo Ramírez.

Correo electrónico: japara18@yahoo.com 